\title{
Calorie-burning fat found in adults
}

The complications of obesity can be life-threatening, but current obesity therapies are limited by adverse effects and only modest efficacy. Now, a trio of studies published in the New England Journal of Medicine report that brown adipose tissue (BAT) - which is capable of using energy to produce heat - is present and functional in adults, making it a potential target for weight loss therapy.

Whereas white adipose tissue (WAT) stores energy, BAT expends energy to generate heat, by uncoupling mitochondrial oxidative phosphorylation through the action of uncoupling protein 1 (UCP1). Although this process has a crucial role in maintaining core body temperature in newborn children, BAT regresses with age, and its presence and physiological relevance in adults has been thought to be minimal. However, various recent studies, using combined positron emission tomography (PET) and computed tomography (CT) techniques to monitor glucose uptake and identify metabolically active tissue, have indicated that active BAT can be present in some adults.

To assess this, Cypess and colleagues studied patient PET-CT scans that had been previously performed for various diagnostic reasons. BAT, identified by its size, density and high uptake of the glucose analogue ${ }^{18} \mathrm{~F}$-fluorodeoxyglucose $\left({ }^{18} \mathrm{~F}-\mathrm{FDG}\right)$, was found in 106 out of 1,972 patients, largely in the cervical and supraclavicular regions. The presence and activity of this tissue correlated inversely with age, body mass index (BMI), fasting plasma glucose levels and treatment with beta-blockers. Intra-individual variation was also noted, with maximal activity apparent in the colder winter months. Active BAT was also more prevalent in women than in men.

The presence of functional adult BAT is further supported by the work of Virtanen and colleagues. In 5 healthy subjects, PET-CT scans revealed that 2 hours of mild cold exposure increased ${ }^{18} \mathrm{~F}$-FDG uptake by tissue in the supraclavicular area by 15 -fold, but not in adjacent WAT. Importantly, histological and gene expression analysis of surgical biopsies confirmed this tissue to be BAT: multilocular lipid droplets were present and mRNA levels of genes that are characteristic of brown fat, including PRDM16 and UCP1, were upregulated compared with those characteristic of WAT. Furthermore, UCP1 was highly expressed and localized to the mitochondria.

Focusing on the influence of body weight on BAT activity, van Marken Lichtenbelt and colleagues studied BAT in 24 healthy lean or overweight men, again using ${ }^{18} \mathrm{~F}-\mathrm{FDG}$ PET-CT. BAT was identified in 23 of the subjects. Following 2 hours of mild cold exposure, this BAT was activated predominantly in the supraclavicular region in all lean and, notably, in 13 out of 14 overweight subjects (although to a lesser extent), revealing the feasibility of targeting this tissue. Activity correlated positively with resting metabolic rate and core body temperature, and correlated negatively with distal skin temperature, BMI and percentage body fat.

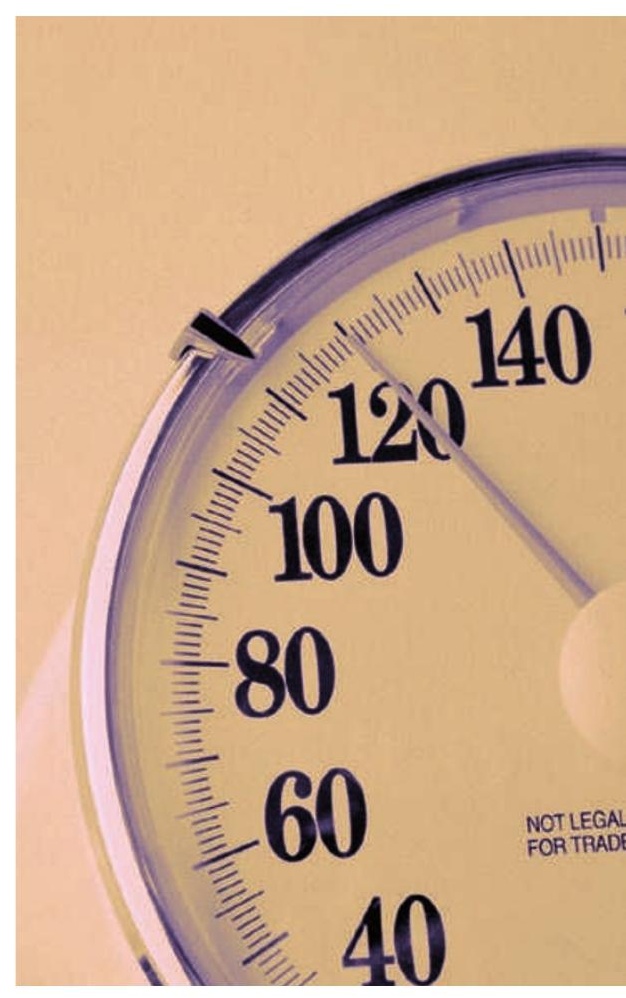

Together, these studies confirm the existence of metabolically active BAT in adults. Given that obesity arises from an imbalance between energy intake and expenditure, the possibility of therapeutically activating BAT warrants further investigation.

Sarah Crunkhorn

ORIGINAL RESEARCH PAPERS van Marken Lichtenbelt, W. D. et al. Cold-activated brown adipose tissue in healthy men. N. Engl.J. Med. 360, 1500-1508 (2009) | Cypess, A. M. et al. Identification and importance of brown adipose tissue in adult humans. N. Engl. J. Med. 360, 1509-1517 (2009) | Virtanen, K. A. et al. Functional brown adipose tissue in healthy adults. N. Engl. J. Med. 360, 1518-1525 (2009) 